\title{
Cardiopatia e gravidez
}

\author{
Pregnancy in patients with heart disease
}

\section{Carolina Burgarelli Testa ${ }^{1}$, Vera Therezinha Medeiros Borges ${ }^{2}$, Maria Rita de Figueiredo Lemos Bortolotto ${ }^{3}$}

Testa CB, Borges VTM, Maria Rita de Figueiredo Lemos Bortolotto MRFL. Cardiopatia e gravidez / Pregnancy in patients with heart disease. Rev Med (São Paulo). 2018 mar.-abr.;97(2):177-86.

RESUMO: A assistência às pacientes cardiopatas passou por enormes avanços nas últimas décadas. Apesar da queda morbidade, a gravidez nesse grupo representa importante causa de mortalidade materna e de pior prognóstico fetal, mesmo em países desenvolvidos. O acompanhamento obstétrico dessas pacientes precisa ser individualizado conforme a estratificação de risco materno. Há poucos ensaios clínicos randomizados, sendo que a maior parte das condutas é baseada em estudos retrospectivos. Neste artigo realizamos uma revisão de literatura para embasar as melhores práticas na assistência multidisciplinar durante o ciclo gravídico puerperal das pacientes portadoras de cardiopatia.

Descritores: Gravidez; Cardiopatias; Complicações cardiovasculares na Gravidez; Cuidado pré-natal.

\section{INTRODUÇÃO}

Aestação em mulheres portadoras de doenças cardíacas sempre esteve associada a altos níveis de morbidade e mortalidade tanto materna quanto perinatal. A sobrecarga imposta pelas modificações hemodinâmicas fisiológicas da gravidez aumentam o risco de descompensação clínica mesmo em pacientes

\begin{abstract}
The care of patients with heart disease has made great strides in recent decades. Despite the reduction in morbidity, pregnancy in this group represents an important cause of maternal mortality and worse fetal prognosis, even in developed countries. Obstetric follow-up of these patients needs to be individualized according to maternal risk stratification. A few randomized clinical trials have been conducted, and most are based on retrospective studies. For this article, we conducted a literature review to support the best practices in multidisciplinary care during the puerperal pregnancy cycle of patients with heart disease.
\end{abstract}

Keywords: Pregnancy; Heart diseases; Pregnancy complications, cardiovascular; Prenatal care.

previamente estáveis. Ao longo do século XX a melhoria da assistência clínica e obstétrica permitiu que a maioria dos casos evoluísse com desfechos favoráveis. Não obstante, a cardiopatia materna continua sendo uma das principais causas de morte materna, tanto em nosso meio quanto em países desenvolvidos ${ }^{1,2}$. O conhecimento da fisiologia e suas implicações sobre a patologia devem nortear a assistência às pacientes com cardiopatia.

1. Hospital das Clínicas HCFMUSP, Faculdade de Medicina, Universidade de São Paulo, São Paulo, SP, BR. ORCID: https://orcid. org/0000-0002-2384-4754. Email: carolina.burgarelli@hc.fm.usp.br.

2. Universidade Estadual Paulista (UNESP), Botucatu, SP, BR. ORCID: https://orcid.org/0000-0002-9347-9641.Email: Vborges0765@ gmail.com.

3. Hospital das Clínicas HCFMUSP, Faculdade de Medicina, Universidade de São Paulo, São Paulo, SP, BR. ORCID: https://orcid.org/ 0000-0001-6674-051X. Email: maria.bortolotto@hc.fm.usp.br.

Endereço para correspondência: Carolina Burgarelli Testa. Avenida Doutor Enéas Carvalho de Aguiar, 255, Instituto Central do HCFMUSP, $10^{\circ}$ andar. CEP 05403-000 São Paulo, SP. Brazil. 


\section{Epidemiologia}

As doenças cardíacas afetam ao redor de $1 \%$ das gestações (as cifras variam de 0,3 a 4,2\%, conforme a população analisada) $)^{3}$. Nos países de alta renda a redução da cardiopatia reumática e o aumento da sobrevivência das mulheres com cardiopatia congênita foram responsáveis pelo predomínio deste tipo de doença dentre as gestantes cardiopatas ${ }^{4}$. Já nos países de baixa e média renda ainda predominam as doenças adquiridas ${ }^{2}$, associadas a maior morbidade ${ }^{1,5,6}$. No período de 2001 a 2005 , de 571 pacientes atendidas na Clínica Obstétrica do Hospital das Clínicas da Universidade de São Paulo, 45,8\% eram portadoras de cardiopatia reumática e $28,6 \%$ cardiopatias congênitas 7 .

No Brasil, Feitosa ${ }^{8}$ reportou prevalência de cardiopatia em 4,2\% das gestações atendidas no Hospital São Paulo entre 1979 e 1998. A cardiopatia foi causa de 6,4\% dos óbitos maternos no Município de São Paulo entre 1993 e 2012 (9), e 8\% dos casos de mortalidade materna no Estado de São Paulo no ano de $2014^{10}$. Outros fatores contribuem para o aumento da gravidade desses casos, como a maternidade tardia e mudanças de estilo de vida (sedentarismo, obesidade, tabagismo), que por sua vez, implicam no aumento da hipertensão arterial e das doenças cardiovasculares ${ }^{11-13}$. Considerando-se que a deterioração clínica materna pode ocorrer no mesmo no puerpério tardio, e que muitos casos de cardiomiopatia periparto se manifestam até 5 meses após o parto, essas cifras podem ser até maiores quando são avaliados os casos de morte materna tardia ${ }^{14,15}$.

\section{Alterações hemodinâmicas gravídicas}

A gestação representa uma grande mudança para todos os órgãos e sistemas. Mudanças fisiológicas progressivas permitem o desenvolvimento fetal e preparam a mãe para o parto. Em mulheres saudáveis, estas alterações não costumam apresentar repercussões clínicas, mas alguns fatores maternos podem influenciar na capacidade adaptativa às demandas gestacionais ${ }^{16}$. Entre as principais adaptações do organismo materno à gestação estão as alterações cardiovasculares ${ }^{17,18}$. A paciente entra em estado hiperdinâmico, com aumentos no débito e na frequência cardíaca, o que põe à prova a reserva funcional cardíaca ${ }^{19}$.

$\mathrm{O}$ volume plasmático materno aumenta em até $10 \%$ entre 6 e 7 semanas de gestação, e atinge valores até $50 \%$ maiores em torno na $32^{\mathrm{a}}$ semana de gravidez, permanecendo estável após. A magnitude do aumento de volemia pode variar conforme o número de fetos ${ }^{20,21}$. A resistência vascular periférica tem sua queda iniciada em estágios precoces da gestação, com seu nadir entre 14 e 24 semanas. A causa primária da queda de resistência vascular periférica é a vasodilatação arterial periférica, mediada desde o início da gestação pela progesterona e óxido nítrico, e com o estabelecimento da circulação uteroplacentária há uma queda mais acentuada ${ }^{4,16,18}$.

Diante da redução da resistência vascular periférica, a pressão arterial sofre redução em torno de $10 \%$ entre 7 e 8 semanas de gestação, com queda progressiva até 24 semanas de gestação e retorna a valores habituais próximo ao termo ${ }^{16}$. Entre os mecanismos fisiológicos regulatórios que compensam esta queda estão o estímulo a barorreceptores arteriais de vasopressina e do sistema renina-angiotensina-aldosterona, que associado à resistência à ação vasoconstritora da angiotensina II, resultam em retenção de sódio e água, com consequente aumento do volume plasmático circulante e manutenção fisiológica do débito cardíaco e da pressão $\operatorname{arterial}^{18}$.

Em resposta a essas alterações, aliadas ao aumento de 20 a 30\% na frequência cardíaca e às alterações anatômicas cardíacas, ocorre aumento compensatório do débito cardíaco, que se inicia ao redor da $7^{\mathrm{a}}$ semana de gestação e aumenta em até $20 \%$ no terceiro trimestre ${ }^{16,17,22-24}$. O débito cardíaco continua aumentando até o fim do segundo trimestre ${ }^{25}$, atingindo valores 30 a $50 \%$ maiores que no estado pré-gestacional e atinge um platô em torno das 32 semanas. Após o parto, existe aumento adicional do débito nos primeiros dias, com retorno à condição prégravídica em até 24 semanas pós-parto ${ }^{26}$.

$\mathrm{O}$ número de fetos influencia igualmente as alterações cardiovasculares, sendo que o débito cardíaco e o volume sistólico em gestações gemelares podem superar em $20 \%$ os valores observados em gestações únicas ${ }^{23,27}$.

Durante o trabalho de parto, as contrações uterinas exercem mais sobrecarga de volume sobre o sistema hemodinâmico materno, promovendo aumento da volemia de até $500 \mathrm{ml}$ e de débito cardíaco de até $30 \%$. A anestesia loco-regional ajuda a amenizar os efeitos de volume e estabilizar a frequência cardíaca ${ }^{16,28}$, além de reduzir o puxo materno (que aumenta momentaneamente o débito) ${ }^{29}$.

Após o parto, ocorre grande reabsorção de fluidos nas primeiras 24 horas, com retorno de aproximadamente $500 \mathrm{ml}$ de sangue da circulação uterina para a circulação, descompressão de veia cava e absorção de terceiro espaço. O débito cardíaco aumenta em até $59 \%$ e o volume ejetado em $71 \%$ no puerpério imediato ${ }^{16,20,30}$. Nesta fase, as pacientes com distúrbios cardiovasculares podem ter dificuldade para a adaptação do débito cardíaco e apresentar insuficiência cardíaca descompensada e /ou edema agudo de pulmão ${ }^{31}$.

\section{Influência das modificações hemodinâmicas gravídicas em gestantes cardiopatas}

As alterações hemodinâmicas fisiológicas previamente descritas sobrecarregam significativamente o sistema cardiovascular da gestante e podem implicar em descompensação clínica grave nas pacientes cardiopatas, inclusive nas previamente assintomáticas, que podem ter 
dificuldades em tolerar o aumento da pré-carga e a redução da pós-carga associadas ao aumento da frequência cardíaca e queda da resistência vascular periférica ${ }^{13}$.

Além disso, no intraparto, o aumento rápido de volume intravascular durante as contrações, bem como o puxo materno associado ao aumento de tônus simpático estimulado pela dor e pela ansiedade são fatores de alto risco para a ocorrência de edema agudo pulmonar. A anestesia peridural pode reduzir o tônus simpático, mas existe risco de hipotensão transitória relacionada à vasodilatação periférica ${ }^{13}$.

As repercussões da gravidez sobre a cardiopatia materna dependem da etiologia da doença, da presença de intervenções cirúrgicas e lesões ou função residual, do tipo de terapêutica empregada, da ocorrência de complicações ou eventos prévios e da condição funcional, sendo que as apresentações clínicas são muito variáveis ${ }^{32-34}$.

Em registro multicêntrico que incluiu 1321 pacientes cardiopatas entre 2007 e 2011, Ruys ${ }^{2}$ encontrou como principal complicação a insuficiência cardíaca (13,1\% dos casos), sendo que a mortalidade materna foi significantemente maior nas pacientes com classe funcional NYHA (New York Heart Association) III ou IV, assim como o óbito fetal e a incidência de prematuridade.

Em geral, as pacientes com cardiopatias congênitas não corrigidas ou com cirurgias paliativas apresentam risco obstétrico elevado, assim como risco fetal e de complicações cardíacas durante a gravidez, mas poucas são as lesões que contraindicam a gestação ${ }^{4}$. As complicações obstétricas, neonatais e cardiovasculares são mais prevalentes nestas pacientes $^{35,36}$ e estão diretamente relacionadas à sua função cardíaca $^{35}$.

Drenthen etal. estudaram 1696 gestações em pacientes com cardiopatias congênitas. Nesta coorte (ZAHARA ZwangerschapbijAangeborenHARtAfwijkingen), houve 19,4\% de abortamentos espontâneos e 3,4\% de interrupções eletivas da gestação, com seguimento de 1302 gestações em 714 pacientes ${ }^{36}$. As complicações perinatais incluíram parto prematuro $(12 \%)$, restrição de crescimento fetal (14\%) e mortalidade neonatal ( 4\%). As complicações maternas foram diretamente relacionadas à presença de cardiopatia cianogênica $(\mathrm{p}<0,0001)$, uso prévio de medicamentos $(\mathrm{p}<0,0001)$, obstrução de via de saída de ventrículo esquerdo $(\mathrm{p}<0,0001)$ e prótese valvar mecânica $(\mathrm{p}=0,0014)^{36}$.

As valvopatias (adquiridas ou congênitas) apresentam grande espectro de lesões, com incidência de complicações clínicas de $15 \%$, entre as quais arritmias, falência ventricular e endocardite. De maneira geral, as lesões regurgitativas são melhor toleradas, e as estenoses implicam em maior repercussão clínica ao longo da gravidez, devido à inabilidade do sistema cardiovascular de se adaptar ao aumento do volume circulante e do débito cardíaco $^{29}$. Portanto, pacientes previamente assintomáticas podem ter sintomas desencadeados e/ou exacerbados diante das modificações gravídicas ${ }^{33,37}$.

$\mathrm{Na}$ estenose mitral grave, ocorre aumento progressivo do volume atrial esquerdo, com aumento de pressão capilar pulmonar e risco de hipertensão pulmonar, sobrecarga e posterior falência de ventrículo direito. O aumento de volume circulante durante a gestação implica diretamente neste mecanismo e pode acarretar em descompensação cardíaca materna ${ }^{29}$. Já na estenose aórtica grave (área valvar $<1 \mathrm{~cm}^{2}$ e gradiente transvalvar $>$ $50 \mathrm{mmHg}$ ) há aumento de pré-carga de ventrículo esquerdo, com possibilidade de hipertrofia ventricular. Durante a gravidez ocorre o aumento do gradiente (redução de pós carga por redução da resistência vascular periférica). As principais complicações podem ocorrer no intraparto, principalmente pela inabilidade em tolerar a redução de pré-carga ${ }^{29}$.

A hipertensão arterial pulmonar é caracterizada por remodelamento vascular, com elevação da pressão média em artéria pulmonar acima de $25 \mathrm{mmHg}$. Pacientes com hipertensão pulmonar são intolerantes às modificações gravídicas e apresentam um risco muito elevado de morbimortalidade ${ }^{33,37}$, com maior chance de falência ventricular direita e deterioração clínica, principalmente no fim do $2^{\circ}$ trimestre e puerpério. A mortalidade materna varia de 30 a $50 \%{ }^{38}$. O controle de débito cardíaco é muito importante, já que as pacientes são dependentes de pré-carga e a redução de débito pode resultar em colapso circulatório.

$\mathrm{O}$ aumento de incidência de coronariopatia em mulheres em idade reprodutiva também é notável. Estimase que o infarto agudo do miocárdio ocorra em 6,2 por 100000 nascimentos. A mortalidade diante de IAM na gestação e puerpério chega a $11 \%$, cm risco materno muito aumentado caso o parto seja realizado durante o quadro agudo e nas duas primeiras semanas após o tratamento, com perda fetal de $9 \%{ }^{33}$.

As obstruções de via de saída (estenose valvares, hipertrofia assimétrica obstrutiva) e disfunções de ventrículo sistêmico limitam o aumento no débito cardíaco e podem restringir a circulação uteroplacentária e o crescimento fetal ${ }^{33}$. Diversos são os estudos que encontraram complicações fetais e/ou neonatais graves (prematuridade, restrição de crescimento fetal, óbito fetal) mais frequentes nas cardiopatas, sendo que este risco é ampliado pela presença de fatores de risco cardíacos como classe funcional NYHA III ou IV, obstrução de via de saída, anticoagulação ou cianose e obstétricos, tais como gemelidade $^{14,39,40}$.

O peptídeo B natriurético (BNP) ou sua porção inativa (NT-proBNP) tem sido proposto como fator preditor de descompensação cardíaca e apresenta ótima ferramenta para estratificação de risco em fases iniciais e durante a gestação. Valores de BNP $<100$ têm um valor preditivo negativo de $100 \%$ para identificar eventos cardiovasculares na gestação ${ }^{13,41}$. Em estudo longitudinal realizado por Blatt et al. ${ }^{41}$ com 7 pacientes com cardiopatia dilatada e fração 
de ejeção de ventrículo esquerdo $<45 \%$, a dosagem de NT-proBNP realizada com 30 semanas de gestação, ao ser comparada com ecocardiograma seriado, apresentou melhor valor prognóstico.

\section{Influência da cardiopatia materna sobre os resultados obstétricos e neonatais}

Diversos são os estudos que relacionam a inadequação das alterações hemodinâmicas a piores resultados obstétricos e perinatais, inclusive em gestantes saudáveis. Borges e colaboradores avaliaram em estudo prospectivo o débito cardíaco através do ecocardiograma em 22 gestantes sem patologias e observaram que as pacientes que tiveram recém-nascidos pequenos para a idade gestacional apresentaram débito cardíaco e diâmetro de átrio esquerdo inalterados ao longo da gestação, associado a $28 \%$ de aumento da resistência periférica ${ }^{42}$. Vasapollo et al. ${ }^{43}$ realizaram um estudo caso-controle com avaliação ecocardiográfica materna em 21 pacientes com gestações únicas e fetos restritos e 21 gestações controle, e encontraram no grupo de fetos restritos menor débito cardíaco, menor fração de ejeção de ventrículo esquerdo e maior resistência vascular periférica.

As repercussões da cardiopatia materna sobre os resultados perinatais dependem também do tipo de cardiopatia, das repercussões clínicas e do tipo de medicamentos empregados ${ }^{44}$. Estudos retrospectivos e prospectivos de gestações em mulheres portadoras de cardiopatia mostram que a ocorrência de eventos perinatais está significativamente aumentada nestes $\operatorname{casos}^{45-47}$, sendo este risco ampliado pela presença de fatores de risco cardíacos e obstétricos ${ }^{48}$. Os principais fatores independentes de influência negativa no prognóstico perinatal são anticoagulação, cianose, classe funcional III ou IV NYHA, gestação múltipla, obstrução de via de saída do ventrículo sistêmico, prótese valvar metálica, tabagismo ${ }^{48,49}$

$\mathrm{Siu}^{49}$ observou prematuridade em $15 \%$ e mortalidade perinatal em $10 \%$ dos casos. Em série de 571 pacientes seguidas na Clínica Obstétrica HCFMUSP (61\% de cardiopatias adquiridas), observamos $1,2 \%$ de óbitos fetais, $28 \%$ de fetos pequenos para a idade gestacional e prematuridade em $22,4 \%$ dos $\operatorname{casos}^{7}$, sendo que a distribuição de fetos de baixo peso foi semelhante entre diferentes etiologias. O principal fator que influenciou a prematuridade foi a classe funcional materna $(25 \% \mathrm{em}$ classe funcional I/II vs $41 \%$ em classe funcional III/IV $)^{7}$.

Gelson et al. ${ }^{40}$ realizaram um estudo de coorte prospectiva com 331 pacientes cardiopatas e 662 controles, e encontraram que o percentil de peso dos recém-nascidos de mães cardiopatas foi menor $(\mathrm{p}<0,001)$, com risco de complicações perinatais maior (34\% vs $15 \%$, OR2,9, $\mathrm{p}<0,001)$. Em análise multivariada, a disfunção miocárdica foi o fator que esteve associado à efeitos adversos perinatais $^{40}$.
Em estudo retrospectivo realizado por Ouyang et al. ${ }^{50}$ em 2010 foram analisadas 112 gestações de 65 mulheres com cardiopatias congênitas em classe funcional 1 ou 2, foram encontrados $32,6 \%$ de eventos obstétricos adversos, sendo o mais frequente o parto prematuro.

Com a finalidade de estudar a placentação em mulheres com cardiopatias congênitas, Pieper et al. ${ }^{35}$, em 2013, realizaram um estudo prospectivo multicêntrico com 209 gestantes portadoras de cardiopatia congênita e 70 gestantes sem patologias, sendo que nenhuma paciente com cardiopatia cianótica não corrigida foi incluída, realizando a avaliação dopplervelocimetrica de artérias uterinas e de artéria umbilical com 20 e 32 semanas de gestação, associado à avaliação de função cardíaca no ecodopplercardiograma e dosagem sérica de NT-proBNP. Entre as características clínicas, a piora de classe funcional ocorreu apenas nas pacientes cardiopatas, das quais $10 \%$ apresentaram eventos cardiovasculares. Os parâmetros prégestacionais e com 20 semanas relacionados à alteração de resistência de artéria umbilical foram a função ventricular direita $(p=0,002)$, dosagem alterada de NT-proBNP $(p=0,085)$, regurgitação valvar sistêmica $(p=0,001)$ ou pulmonar $(\mathrm{p}=0,045)$. Os eventos obstétricos ocorreram em 58,9\% das pacientes com cardiopatia congênita $v s$. $32,9 \%$ nas pacientes saudáveis, $\mathrm{p}<0,005$, e os partos vaginais assistidos também foram mais prevalentes (47,4\% vs. $25,7 \%, p=0,001)$. Em pacientes cardiopatas, o aumento de resistência da artéria umbilical com 32 semanas foi relacionado aos eventos obstétricos $(\mathrm{p}=0,049)$, apresentaram menor idade gestacional no parto (38,3 vs. $39,7$ semanas, $\mathrm{p}<0,005)$ e seus recém-nascidos tiveram menor peso de nascimento ( $3036 \mathrm{~g}$ vs. $3578 \mathrm{~g}, \mathrm{p}<0,005$ ) e mais frequentemente Apgar $<9$ no $10^{\circ}$ minuto $(8,7 \%$ vs. $0 \%$, $\mathrm{p}=0,009)$. Os autores concluíram que os parâmetros de fluxo placentário estão alterados nas pacientes com cardiopatias congênitas e sugerem que este fator possa implicar em uma maior incidência de complicações obstétricas neste grupo ${ }^{35}$.

\section{Avaliação de Risco}

Para a estratificação de risco devemos levar em conta as alterações hemodinâmicas fisiológicas da gravidez e as patologias cardíacas com alta taxa de mortalidade. A estratificação de risco determinará a frequência de reavaliação clínica durante a gravidez e também auxiliará no trabalho de parto, na escolha do tipo de parto e na avaliação pós-parto.

\section{Preditores de risco cardíaco materno}

Inúmeros estudos têm sido realizados com o intuito de identificar e estratificar o risco destas pacientes. Dentre eles podemos destacar o obtido pelo o estudo de coorte, prospectivo e multicêntrico (CARPREG), realizado por Siu et al. ${ }^{49} \mathrm{em}$ gestantes com doença cardíaca. O estudo incluiu $75 \%$ mulheres com doença congênita e $25 \%$ de doença 
adquirida e excluíram aquelas com prolapso de valva mitral sem insuficiência. A frequência de complicações cardiovasculares durante a gestação foi de $13 \%$, metade durante o parto, sendo que as principais foram insuficiência cardíaca e arritmias. Foram registradas três mortes. Neste estudo foram definidos quatro fatores preditores de complicações maternas: classe funcional (CF) NYHA > II antes da gravidez; cianose; disfunção ventricular esquerda moderada/grave $(\mathrm{FE}<40 \%)$; obstrução do coração esquerdo (área valvar mitral $<2 \mathrm{~cm}^{2}$, área valvar aórtica $<$ $1,5 \mathrm{~cm}^{2}$, gradiente de pressão da artéria pulmonar média $>30 \mathrm{mmHg}$ ) e antecedentes de eventos cardiovasculares (tromboembolismo, arritmias, endocardite infecciosa, insuficiência cardíaca). O risco estimado de complicações durante a gravidez em pacientes com doença cardíaca, sem nenhum fator preditor descrito acima, foi de 5\%; com 1 fator, 27\%; e com 2 fatores, $75 \%$.

Outra classificação de risco utilizada foi elaborada pela Organização Mundial da Saúde (OMS), que com a colaboração de programas internacionais de planejamento familiar e por meio da revisão sistemática da literatura, elaborou documentos recomendando o método mais adequado de contracepção nas mulheres cardiopatas, definindo também os riscos da gravidez nesta população ${ }^{51-53}$. Essa classificação de risco foi dividida em quatro categorias:

- Classe 1: risco de morbidade e mortalidade semelhante à da população geral. As patologias cardíacas incluídas são: valvopatias com lesões leves, cardiopatia congênita corrigida e sem sequela (exemplos - comunicação atrial, persistência do canal arterial), prolapso de valva mitral competente, presença de extrassístoles ventriculares isoladas. O acompanhamento da cardiologia durante a gravidez pode ser limitado a uma ou duas visitas;

- Classe 2: risco levemente aumentado de morbidade e mortalidade materna. Nesta categoria encontramos: tetralogia de Fallot corrigida, defeitos septais não corrigidos e a maioria das arritmias. Recomenda-se o acompanhamento da cardiologia em cada trimestre;

- Classe 2-3: dependendo das circunstâncias individuais as pacientes podem ser classe 2 ou 3 . O risco está aumentado se houver fatores de risco adicionais ou uma combinação de condições. Por exemplo, o risco de gravidez em uma mulher com insuficiência mitral e aórtica moderada e função ventricular discretamente alterada será a classe 3. Exemplos dessa classe: disfunção ventricular leve, cardiomiopatia hipertrófica, síndrome de Marfan sem dilatação aórtica, disfunção ventricular leve a moderada, estenose pulmonar grave, miocardiopatia periparto prévia sem disfunção ventricular residual;

- Classe 3: aumento significativo do risco de morbidade ou mortalidade materna. Exemplos desta categoria são: próteses valvares cardíacas mecânicas, transposição de grandes vasos corrigidas, coarctação da aorta não corrigida e válvula aórtica bicúspide com aorta ascendente com diâmetro menor que $45 \mathrm{~mm}$, cardiopatia cianótica, outras cardiopatias congênitas complexas. Essas mulheres necessitam aconselhamento pré-concepção e cuidados durante o pré-natal e parto, com avaliação cardiológica mensal. Pode ser necessária internação para observação e compensação clínica;

- Classe 4: elevada morbidade e mortalidade, portanto a gravidez está contraindicada e caso ocorra deve ser proposto aborto terapêutico. Se a gravidez continuar os cuidados são semelhantes as pacientes da classe 3 . As patologias incluídas nesta categoria são: hipertensão pulmonar de qualquer causa, disfunção ventricular sistêmica grave, insuficiência cardíaca grau III-IV ou fração de ejeção menor que $30 \%$, miocardiopatia periparto em gestação anterior com disfunção ventricular, síndrome de Marfan com dilatação da aorta maior que $40 \mathrm{~mm}$ e estenose aórtica grave.

\section{Preditores de risco fetal}

As mulheres com cardiopatia apresentam taxas de complicações fetais e neonatais maiores do que na população geral, e as mais frequentes são: restrição de crescimento fetal, prematuridade, hemorragia intracraniana e óbito fetal.

$\mathrm{O}$ estudo de Siu et al. ${ }^{49}$ encontrou incidência de $20 \%$ de complicações neonatais, sendo as mais frequentes restrição de crescimento intrauterino e prematuridade. Os fatores preditores destas complicações foram os mesmos citados para risco materno, além do uso de anticoagulante, gestação gemelar, tabagismo e idade materna menor que 20 anos ou maior que 35 anos. No estudo ZAHARA os preditores independentes de risco obstétrico descritos foram a presença de próteses valvares mecânicas, cardiopatia cianótica, uso de medicação cardiovascular prévio à gravidez e ainda fatores não cardiológicos (tabagismo e gemelaridade). É interessante observar que nesses estudos a presença de hipertensão pulmonar não foi preditora de complicações obstétricas nem cardíacas, provavelmente porque está sub-representada na população estudada (europeia e canadense). No estudo de Ruys ${ }^{2}$ (ROPAC - the Registry on Pregnancy and Cardiac Disease), compreendendo registro multicêntrico de 28 países, muitos em desenvolvimento (onde a hipertensão pulmonar é mais prevalente), foi observado que a hipertensão pulmonar é um fator independente de insuficiência cardíaca precoce na gravidez (antes da $30^{\mathrm{a}}$ semana), o que pode ter determinado mais partos prematuros terapêuticos.

$\mathrm{Na}$ série de pacientes analisadas no estudo ROPAC $^{2,54}$ foi observada relação da classificação da OMS com os eventos maternos e perinatais (Tabela 1). Pode-se observar que a ocorrência de eventos adversos foi mais intensa conforme a classe à qual as pacientes pertenciam. 
Testa CB, et al. Cardiopatia e gravidez.

Tabela 1 - distribuição dos eventos maternos e perinatais de acordo com as categorias de risco da OMS

\begin{tabular}{l|c|c|c|c|c|c}
\hline & TOTAL & $\begin{array}{c}\text { OMS } \\
\text { classe I }\end{array}$ & $\begin{array}{c}\text { OMS } \\
\text { classe II }\end{array}$ & $\begin{array}{c}\text { OMS } \\
\text { classe III }\end{array}$ & $\begin{array}{c}\text { OMS } \\
\text { classe IV }\end{array}$ & P \\
\hline Mortalidade materna & $1 \%$ & 0 & $1 \%$ & $1 \%$ & $4 \%$ & 0,086 \\
\hline Internação materna & $26 \%$ & $13 \%$ & $18 \%$ & $36 \%$ & $66 \%$ & $<0,001$ \\
\hline Insuficiência cardíaca & $12 \%$ & $1 \%$ & $6 \%$ & $19 \%$ & $57 \%$ & $<0,001$ \\
\hline Cesáreas & $41 \%$ & $27 \%$ & $37 \%$ & $49 \%$ & $60 \%$ & $<0,001$ \\
\hline Hemorragia pós-parto & $3 \%$ & 0 & $1 \%$ & $5 \%$ & $11 \%$ & $<0,001$ \\
\hline Parto $<37$ semanas & $15 \%$ & $9 \%$ & $15 \%$ & $17 \%$ & $30 \%$ & $<0,001$ \\
\hline Óbito fetal & $2 \%$ & 0 & $1 \%$ & $3 \%$ & $6 \%$ & 0,001 \\
\hline Óbito neonatal & $1 \%$ & $1 \%$ & 0 & 0 & 0 & 0,5 \\
\hline Média Peso RN (gramas) & 3010 & 3109 & 3074 & 2925 & 2735 & $<0,001$ \\
\hline $\mathrm{N}$ & 1321 & 241 & 514 & 504 & 53 & - \\
\hline
\end{tabular}

FONTE: Adaptado de Ruys et al. ${ }^{54}$

Devemos ainda lembrar que gestantes portadoras de cardiopatias congênitas apresentam risco maior de terem filhos cardiopatas. $\mathrm{O}$ risco de cardiopatia congênita na população geral é de 0,8 a $1 \%$, chegando até a $12 \%$ quando um dos pais é portador da doença. Em casos específicos pode chegar até a 50\% - síndromes de DiGeorge, Marfan, Noonan, ou ainda na cardiomiopatia hipertrófica (forma familiar) $)^{39,44,45}$.

\section{Assistência Pré-Natal}

A equipe de assistência à gestante cardiopata deve ser multidisciplinar, composta por obstetras, cardiologistas, anestesiologistas, pediatras, enfermagem, psicólogos, nutricionistas, fisioterapeutas eassistentes sociais aptos a atender este público. Preferencialmente esta assistência deve ser realizada em centro de atendimento terciário ou até quaternário. Presume-se que a avaliação inicial deve ser realizada o mais precoce possível e durante esta consulta inicial devemos conscientizar a paciente sobre os riscos previsíveis da gravidez e da importância do seguimento das orientações e do acompanhamento frequente.

As condutas preconizadas segundo diretrizes nacionais $^{55,56}$, internacionais ${ }^{52}$ e protocolos locais $^{34}$ são:

- Controle de peso materno: é importante para minimizar a sobrecarga cardíaca, que não ocorra ganho de peso exagerado e ocasionalmente pode haver a necessidade de realizar dieta hipossódica para controle da retenção hídrica;

- Repouso: estará na dependência da capacidade funcional do coração, segundo a classificação do New York Heart Association (NYHA), lembrando que a classificação pode ser enganosa, pois muitas pacientes podem, inconscientemente, reduzir seu esforço físico dentro dos limites de sua disfunção e negar qualquer incapacidade;

- Evitar tabagismo;

- Suplementação de ferro: avaliar cuidadosamente a necessidade de reposição de ferro, uma vez que a anemia aumenta o trabalho cardíaco;

- Detecção e tratamento precoce de infecções (processos infecciosos também aumentam o trabalho cardíaco);

- Terapêutica medicamentosa cardiovascular: deve ser reavaliada, ajustando a dose e substituindo drogas que possam prejudicar o concepto, como é o caso do anticoagulante oral (no período de embriogênese), inibidores das enzimas conversoras de angiotensina II, bloqueadores dos receptores da angiotensina II e hidantoinatos. Estatinas e espironolactona também estão contraindicadas; o emprego de varfarina deve ser judicioso, reservado aos casos de pacientes com próteses valvares mecânicas ou fibrilação atrial crônica com alto risco trombótico. A amiodarona deve ser evitada (pelo risco de hipotireoidismo neonatal), devendo ser utilizada apenas para arritmias graves que não respondam a outras drogas. Praticamente todos os outros medicamentos cardiovasculares podem ser administrados, se o benefício superar o eventual risco;

- Prevenção da doença reumática: está indicada nas pacientes com valvopatia reumática. Deve ser administrada penicilina benzatina - 1.200.000 UI cada 21 dias, durante toda gestação, pelo risco de novos surtos reumáticos durante este período;

- Profilaxia para endocardite bacteriana (EI): ainda que a antibioticoprofilaxia para EI seja questionada em procedimentos obstétricos não complicados, recomendamos essa medida, uma vez que na prática cotidiana de obstetrícia nem sempre se pode antecipar complicações ou contaminação. Está indicada antes de curetagem pós aborto, partos vaginais, cesarianas e procedimentos obstétricos invasivos, em pacientes de risco moderado a alto para EI (valvopatias, portadoras de próteses valvares, cardiopatias congênitas complexas ou com shunts cirúrgicos sistêmicopulmonares ou qualquer paciente com antecedente de EI), conforme esquema abaixo: 
○ Ampicilina $2 \mathrm{~g}$ por via intravenosa e gentamicina $1,5 \mathrm{mg} / \mathrm{kg}$ (até $120 \mathrm{mg}$ ) antes do procedimento;em casos de alergia a penicilina e derivados - vancomicina $1 \mathrm{~g}$ por via intravenosa (diluída em $100 \mathrm{ml}$ de SF, infundir em uma hora);

○ Para procedimentos dentários, endoscopia, procedimentos em tecidos infectados - amoxicilina $2 \mathrm{~g}$ por via oral, uma hora antes do procedimento. Em caso de alergia a derivados de penicilina - clindamicina $600 \mathrm{mg}$ ou azitromicina $500 \mathrm{mg}$.

- Inibição do trabalho de parto prematuro: inicialmente devemos observar se o trabalho de parto não é decorrente de uma descompensação cardíaca. Frequentemente, com a melhora da condição clínica e da perfusão tissular as contrações reduzem e até cessam. Em casos de baixa reserva funcional e obstruções de fluxo (estenoses valvares, difunção ventricular moderada ou grave) o uso de tocolíticos é contraindicado, pelo risco de piora do quadro cardiovascular. Nas outras situações podese empregar judiciosamente o atosibano, sempre em locais com retaguarda de terapia intensiva (pelo risco de edema agudo pulmonar associado à tocólise e uso de corticóides para maturação pulmonar fetal);

- Corticoterapia para maturação pulmonar fetal - pode provocar sobrecarga de volume e precipitar ou piorar insuficiência cardíaca. Não deve ser empregada rotineiramente. Recomenda-se uso judicioso, sempre em ambiente hospitalar com suporte clínico adequado ${ }^{34}$;

- Prevenção do tromboembolismo: as situações cardiológicas mais frequentemente exigem terapêutica anticoagulante na gravidez são: próteses mecânicas; fibrilação atrial; antecedentes de tromboembolismo; trombo intracavitário; insuficiência cardíaca congestiva (ICC); risco de trombose arterial ou venosa; risco de embolia pulmonar.

O esquema mais apropriado de anticoagulação nas pacientes com próteses valvares mecânicas ainda não está estabelecido. Propostas alternativas têm sido cogitadas, sendo o mais aceito ${ }^{34,44}$.

- Primeiro trimestre: utilizar heparina subcutânea (manter o TTPA duas vezes do valor basal) ou heparina de baixo peso molecular $-1 \mathrm{mg} / \mathrm{Kg}-12 / 12 \mathrm{~h}$. Neste caso, recomenda-se a monitoração com atividade heparínica (ou antiXa entre 0,6 e 1,0);

- Da $13^{\mathrm{a}}$ até $36^{\mathrm{a}}$ semana da gestação, usar anticoagulante oral - dose de acordo a com International Normatization Relation (INR), manter INR $=2,5$ a 3,5. O controle da anticoagulação oral deve ser mais frequente durante a gravidez, devido ao aumento dos fatores prócoagulantes ao longo da gestação, e também devido ao risco de hemorragia fetal em caso de INR elevado;

- Entre a $34^{\mathrm{a}}$ e $36^{\mathrm{a}}$ semana: retornar para heparina e programar o parto. Na indução de parto utilizar heparina intravenosa e interromper a infusão quando a paciente entrar em trabalho de parto. Reintroduzir heparina 6 a 12 horas após o parto;

- No puerpério, fazer a transição para anticoagulação oral assim que houver segurança em relação a sangramento perioperatório, suspendendo a heparina (regular ou de baixo peso molecular) quando o INR estiver na faixa adequada.

$\mathrm{Na}$ fibrilação atrial existe tendência a adotar a mesma conduta, apesar de não existirem estudos em população de gestantes. Nos outros casos citados pode ser utilizada a heparina de baixo peso molecular (ou até heparina regular) em doses profiláticas ou terapêuticas (discutir caso a caso com a equipe de cardiologia).

Exames complementares: devem ser solicitados exames complementares para o diagnóstico e acompanhamento da cardiopatia, como as provas de função reumática, eletrocardiograma, ecocardiograma, e outros conforme a necessidade de cada situação clínica.

Procedimentos cardiológicos: se houver indicação, podem ser realizadas cardioversão elétrica ou medicamentosa, e até implante de marcapasso. A valvoplastia e a cirurgia cardíaca, quando necessárias, deverão ser programadas para ser realizadas entre 13-28 semana, período mais favorável para a mãe e o feto. Devido ao alto risco de morbidade fetal e materna, a cirurgia na gravidez só é recomendada em casos refratários ao tratamento clínico.

Marcação de retornos: depende da gravidade da lesão cardíaca. Pacientes das classes 3 e 4 da OMS podem necessitar de retornos mais frequentes ou até internações prolongadas para vigilância clínica materna e da vitalidade fetal.

\section{Assistência ao parto e puerpério}

As decisões em relação à época de resolução da gravidez e via de parto em mulheres portadoras de cardiopatia ainda são objeto de debates na literatura ${ }^{57,58}$. Quanto à época do parto, a conduta dependerá das condições maternas e fetais. Em pacientes sem repercussão hemodinâmica e boa evolução fetal, deve-se chegar ao termo, preferencialmente aguardando o trabalho de parto espontâneo. Já em pacientes do grupo Classe 3 da OMS, com doenças mais graves ou de maior repercussão hemodinâmica, recomenda-se antecipação do parto (a partir da $37^{\mathrm{a}}$ semana). A indução do parto pode ser indicada por motivos fetais (restrição de crescimento, por exemplo) ou por motivos maternos (piora das condições clínicas), ou ainda para proporcionar melhor apoio logístico e terapêutico em alguns casos - manejo de anticoagulantes, disponibilidade de vaga em terapia intensiva no puerpério ${ }^{34}$. Nos casos de pacientes com repercussão clínica importante, em insuficiência cardíaca refratária ao tratamento clínico, o parto estará indicado, independente da idade gestacional $^{34,52,56}$. 
Em relação à via de parto, existe tendência à indicação liberal de cesarianas nessa população (mesmo sem evidências), por medo das alterações hemodinâmicas ligadas ao trabalho de parto e período expulsivo ${ }^{58}$. Entretanto, em cesáreas existe maior risco de perda sanguíneas, infecções e fenômenos tromboembólicos, o que devem ser levados em conta no momento de sua indicação. Excetuando-se algumas poucas situações, a via de parto é indicada pelas condições obstétricas ${ }^{52,56,58}$. As indicações de cesárea por motivos cardiológicos estão reservadas às pacientes com risco de dissecção de aorta (coarctação de aorta, ou síndrome de Marfan com diâmetro de aorta ascendente superior a $40 \mathrm{~mm}$ ), e em pacientes sob anticoagulação oral no momento do parto (pelo risco de sangramento cerebral fetal decorrente dos fenômenos plásticos do parto). Constituem indicações relativas de cesárea estenose aórtica grave, infarto do miocárdio recente, hipertensão pulmonar grave e insuficiência cardíaca descompensada. Nestes casos, devem ser considerados os riscos maternos, duração do parto, recursos disponíveis, e ainda condição fetal $^{34,56,58}$.

A analgesia neuraxial promove redução da sobrecarga hemodinâmica decorrente do trabalho de parto, e é recomendada em todas as pacientes com cardiopatia, devendo ser indicada precocemente ${ }^{16,28}$. Durante o trabalho de parto, a paciente deve ser mantida em posição lateral (para facilitar o retorno venoso), ou ainda em decúbito elevado $^{34}$. A administração de líquidos deve ser reduzida e monitorada, principalmente durante a indução, devido ao efeito antidiurético da ocitocina ${ }^{52,55}$. A monitoração materna deve ser cuidadosa, com ausculta cardíaca, pulmonar, controle de diurese, cardioscopia e oximetria e pressão arterial. Em casos de maior instabilidade hemodinâmica pode-se recorrer ao controle intra-arterial da pressão; monitoração com cateteres centrais são raramente indicados nos dias atuais ${ }^{29,59}$. No momento do período expulsivo, recomenda-se abreviação com fórcipe ou vácuo-extrator nas pacientes com repercussão hemodinâmica, para evitar as oscilações do débito cardíaco relacionadas aos puxos. O risco de sangramento é aumentado nos partos de pacientes cardiopatas, especialmente nas que recebem anticoagulantes, nas cianóticas e naquelas com hipertensão pulmonar. Recomenda-se hemostasia rigorosa e cuidados

\section{REFERÊNCIAS}

1. Campanharo FF, Cecatti JG, Haddad SM, Parpinelli MA, Born D, Costa ML, et al. The impact of cardiac diseases during pregnancy on severe maternal morbidity and mortality in Brazil. PLoS One. 2015;10(12):e0144385. doi: 10.1371/ journal.pone.0144385.

2. Ruys TPE, Roos-Hesselink JW, Hall R, Subirana-Domènech MT, Grando-Ting J, Estensen M, et al. Heart failure in pregnant women with cardiac disease: data from the ROPAC. Heart. 2014;100(3):231-8. doi: 10.1136/heartjnl-2013-304888. com hipotonia uterina. O emprego de alcalóides do ergot e ocitocina em "bolus" estão contra-indicados; na necessidade de mais agentes uterotônicos, prefere-se o misoprostol por via retal ${ }^{34,52,56}$.

Se nos partos vaginais a analgesia loco-regional é amplamente recomendada, nas cesarianas de pacientes com reserva hemodinâmica limitada, na dependência da condição clínica materna, urgência do procedimento e experiência do anestesista a anestesia geral pode ser indicada. Independente da via de parto, nas pacientes anticoaguladas as anestesias neuraxiais estão contraindicadas. O manejo periparto da anticoagulação visa reduzir os riscos anestésicos e de sangramento materno. As heparinas devem ser suspensas de 12 a 24 horas antes do procedimento (dependendo da dose utilizada), e reintroduzida até 12 horas após do parto ${ }^{34}$.

No puerpério a atenção deve ser redobrada; cerca de dois terços dos óbitos maternos relacionados à cardiopatia ocorrem nesse período ${ }^{8}$. O aumento da volemia nos primeiros dias após o parto pode precipitar descompensação clínica, além dos riscos associados à ocorrência de anemia, tromboembolismo e infecção. As pacientes devem receber cuidados intensivos nas primeiras 24 ou 48 horas após o parto, e a alta deve ser tardia. A profilaxia antitrombótica deve ser considerada em todas as pacientes com cardiopatia, mesmo nas que não faziam uso antes do parto, pelo maior risco de tromboembolismo no puerpério. $\mathrm{O}$ ajuste da terapêutica medicamentosa é feito inicialmente em ambiente hospitalar, com mais opções, principalmente dos inibidores da enzima de conversão e bloqueadores dos receptores da angiotensina II (permitidos nessa fase, mesmo em nutrizes), e emprego mais liberal de diuréticos. A alta só será dada quando a paciente estiver estabilizada ${ }^{34,52,55,56}$. No puerpério tardio, deve ser recomendada contracepção adequada ao período e à condição clínica da paciente.

Em suma, a gestação em paciente portadora de cardiopatia representa um desafio hemodinâmico, com repercussões maternas e perinatais importantes. A assistência adequada evita complicações mais graves, e deve ser oferecida por equipe multiprofissional, em locais com recursos adequados, visando redução da morbiletalidade do binômio materno-fetal.

3. Franklin WJ, Gandhi M. Congenital heart disease in pregnancy. Cardiol Clin. 2012;30(3):383-94. doi: 10.1016/j. ccl.2012.04.003.

4. Rao S, Ginns JN. Adult congenital heart disease and pregnancy. Semin Perinatol. 2014;38(5):260-72. doi: 10.1053/j.semperi.2014.04.015.

5. Abdel-Hady ES, El-Shamy M, El-Rifai AA, Goda H, AbdelSamad A, Moussa S. Maternal and perinatal outcome of 
pregnancies complicated by cardiac disease. Int J Gynaecol Obstet. 2005;90(1):21-5. doi: 10.1016/j.ijgo.2005.03.008.

6. Ruys TPE, Cornette J, Roos-Hesselink JW. Pregnancy and delivery in cardiac disease. J Cardiol. 2013;61(2):107-12. doi: 10.1016/j.jjcc.2012.11.001.

7. Bortolotto MRL. Estudo dos fatores relacionados à determinação da via de parto em gestantes portadoras de cardiopatias. Universidade de São Paulo; 2005.

8. Feitosa HN, Moron AF, Born D, Almeida PAM. Mortalidade materna por cardiopatia. Rev Saúde Publica. 1991;25(6):44351. Disponível em: https://www.scielosp.org/article/ $\mathrm{ssm} /$ content/raw/?resource_ssm_path=/media/assets/rsp/ v25n6/05.pdf .

9. Comitê Central de Mortalidade Materna do Município de São Paulo. Relatório do estudo de casos de mortalidade materna no município de São Paulo durante o ano de 2000. Disponível em: http://www.prefeitura.sp.gov.br/cidade/secretarias/ upload/saude/arquivos/mulher/RelMortMaterna00.pdf.

10. Painel de monitoramento de Mortalidade Materna por CID 10. Datasus. 2015. Disponível em: http://svs.aids.gov.br/ dantps/centrais-de-conteudos/paineis-de-monitoramento/ mortalidade/materna/.

11. Jastrow N, Meyer P, Khairy P, Mercier LA, Dore A, Marcotte $\mathrm{F}$, et al. Prediction of complications in pregnant women with cardiac diseases referred to a tertiary center. Int J Cardiol. 2011;151(2):209-13. doi: 10.1016/j.ijcard.2010.05.045.

12. Deneux-Tharaux C, Berg C, Bouvier-Colle M-H, Gissler M, Harper M, Nannini A, et al. Underreporting of pregnancyrelated mortality in the United States and Europe. Obstet Gynecol. 2005;106(4):684-92. doi: 10.1097/01. AOG.0000174580.24281.e6.

13. Lewey J, Haythe J. Cardiomyopathy in pregnancy. Semin Perinatol. 2014;38(5):309-17. doi: 10.1053/j. semperi.2014.04.021.

14. Grewal J, Siu SC, Ross HJ, Mason J, Balint OH, Sermer $\mathrm{M}$, et al. Pregnancy outcomes in women with dilated cardiomyopathy. J Am Coll Cardiol. 2009;55(1):45-52. doi: 10.1016/j.jacc.2009.08.036.

15. Balint OH, Siu SC, Mason J, Grewal J, Wald R, Oechslin $\mathrm{EN}$, et al. Cardiac outcomes after pregnancy in women with congenital heart disease. Heart. 2010;96(20):1656-61. doi: 10.1136/hrt.2010.202838.

16. Carlin A, Alfirevic Z. Physiological changes of pregnancy and monitoring. Best Pract Res Clin Obstet Gynaecol. 2008;22(5):801-23. doi: 10.1016/j.bpobgyn.2008.06.005.

17. Savu O, Jurcut R, Giusca S, van Mieghem T, Gussi I, Popescu $\mathrm{B}$ a., et al. Morphological and functional adaptation of the maternal heart during pregnancy. Circ Cardiovasc Imaging. 2012;5(3):289-97. doi: 10.1161/CIRCIMAGING.111.970012.

18. St-Louis J, Brochu M. The cardiovascular paradox of pregnancy. Med Sci (Paris). 2007;23(11):944-9. doi: 10.1051/ medsci/20072311944.

19. Zentner D, du Plessis M, Brennecke S, Wong J, Grigg L, Harrap SB. Deterioration in cardiac systolic and diastolic function late in normal human pregnancy. Clin Sci (Lond).

\section{9;116(7):599-606. doi: 10.1042/CS20080142.}

20. Norwitz E, Robinson J, Malone F. Pregnancy-induced physiologic alterations. In: Dildy III G, Belfort M, Saade G, Phelan J, Clark SL, editors. Critical care obstetrics. New Jersey: Blackwell; 2004. Chap.4, p.30-52. https://doi. org/10.1002/9781444316780.ch4.

21. van Oppen AC, Stigter RH, Bruinse HW. Cardiac output in normal pregnancy: a critical review. Obstet Gynecol. 1996;87(2):310-8. doi: 10.1016/0029-7844(95)00348-7.

22. Bamber JH, Dresner M. Aortocaval compression in pregnancy: the effect of changing the degree and direction of lateral tilt on maternal cardiac output. Anesth Analg. 2003;97(1):256-8.

23. Kametas N. Maternal cardiac function in twin pregnancy. Obstet Gynecol. 2003;102(4):806-15.

24. Mahendru AA, Everett TR, Wilkinson IB, Lees CC, McEniery CM. Maternal cardiovascular changes from pre-pregnancy to very early pregnancy. J Hypertens [Internet]. 2012 Nov [cited 2013 Dec 25];30(11):2168-72.

25. Turan OM, De Paco C, Kametas N, Khaw A, Nicolaides $\mathrm{KH}$. Effect of parity on maternal cardiac function during the first trimester of pregnancy. Ultrasound Obstet Gynecol. 2008;32(7):849-54. doi: 10.1002/uog.5354.

26. Bamfo JE a K, Kametas N a, Nicolaides KH, Chambers JB. Maternal left ventricular diastolic and systolic longaxis function during normal pregnancy. Eur J Echocardiogr. 2007;8(5):360-8. doi: 10.1016/j.euje.2006.12.004.

27. Van Mieghem T, Deprest J, Verhaeghe J. Fetal and maternal hemodynamics in pregnancy: new insights in the cardiovascular adaptation to uncomplicated pregnancy, twin-to-twin transfusion syndrome and congenital diaphragmatic hernia. Facts Views Vis Obgyn. 2011;3(3):205-13. Available from: https://www.ncbi.nlm.nih.gov/pmc/articles/PMC3991459/.

28. Tihtonen K, Kööbi T, Yli-Hankala A, Uotila J. Maternal hemodynamics during cesarean delivery assessed by wholebody impedance cardiography. Acta Obstet Gynecol Scand. 2005;84(4):355-61. doi: 10.1111/j.0001-6349.2005.00489.x.

29. Kuczkowski KM, van Zundert A. Anesthesia for pregnant women with valvular heart disease: the state-of-the-art. J Anesth. 2007;21(2):252-7. doi: 10.1007/s00540-007-0504-3.

30. Duvekot JJ, Peeters LL. Maternal cardiovascular hemodynamic adaptation to pregnancy. Obstet Gynecol Surv. 1994;49(12 Suppl):S1-14.

31. Hegewald MJ, Crapo RO. Respiratory physiology in pregnancy. Clin Chest Med. 2011;32(1):1-13, vii. doi: 10.1016/j.ccm.2010.11.001.

32. Zugaib M. Obstetrícia. 2a ed. São Paulo: Manole; 2012.

33. Curry R, Swan L, Steer PJ. Cardiac disease in pregnancy. Curr Opin Obstet Gynecol. 2009;21(6):508-13. doi: 10.1097/ GCO.0b013e328332a762.

34. Bortolotto MRFL, Testa C. Cardiopatias. In: Zugaib M, Bittar RE, Francisco R, editores. Protocolos assistenciais - Clínica Obstétrica FMUSP. 5a ed. São Paulo: Atheneu; 2016. p.181-96.

35. Pieper PG, Balci A, Aarnoudse JG, Kampman M a M, Sollie $\mathrm{KM}$, Groen H, et al. Uteroplacental blood flow, cardiac function, and pregnancy outcome in women with congenital 
heart disease. Circulation. 2013;128(23):2478-87. doi: 10.1161/CIRCULATIONAHA.113.002810.

36. Drenthen W, Boersma E, Balci A, Moons P, Roos-Hesselink JW, Mulder BJM, et al. Predictors of pregnancy complications in women with congenital heart disease. Eur Heart J. 2010;31(17):2124-32. doi: 10.1093/eurheartj/ehq200.

37. Stout K, Otto C. Pregnancy in women with valvular heart disease. Heart. 2007;93(5):552-8. doi: 10.1136/ hrt.2005.067975.

38. Safdar Z. Pulmonary arterial hypertension in pregnant women. Ther Adv Respir Dis. 2013;7(1):51-63. doi: $10.1177 / 1753465812461680$.

39. Uebing A, Steer PJ, Yentis SM, Gatzoulis MA. Pregnancy and congenital heart disease. BMJ. 2006;332(7538):401-6. doi: $10.1136 /$ bmj. 332.7538 .401

40. Gelson E, Curry R, Gatzoulis M a, Swan L, Lupton M, Steer P, et al. Effect of maternal heart disease on fetal growth. Obstet Gynecol. 2011;117(4):886-91. doi: 10.1097/ AOG.0b013e31820cab69.

41. Blatt A, Svirski R, Morawsky G, Uriel N, Neeman O, Sherman $\mathrm{D}$, et al. Short and long-term outcome of pregnant women with preexisting dilated cardiomypathy: an NTproBNP and echocardiography-guided study. Isr Med Assoc J. 2010;12(10):613-6. Available from: https://www.ima.org.il/ FilesUpload/IMAJ/0/40/20199.pdf.

42. Borges VTM, Matsubara B, Peraçoli JC, Calderon IMP, Abbade JF, Rudge MVC. Influência das alterações hemodinâmicas maternas sobre o desenvolvimento fetal. Rev Bras Ginecol Obs. 2001;23(3):147-51. http://dx.doi. org/10.1590/S0100-72032001000300003.

43. Vasapollo B, Valensise H, Novelli GP, Larciprete G, Di Pierro $\mathrm{G}$, Altomare $\mathrm{F}$, et al. Abnormal maternal cardiac function and morphology in pregnancies complicated by intrauterine fetal growth restriction. Ultrasound Obstet Gynecol. 2002;20(5):452-7. doi: 10.1046/j.1469-0705.2002.00847.x.

44. Testa, CB; Bortolotto MRFL, Francisco R. In: Martins MA, Flair JC, Alves VAF, Castilho EA, Cerri G, editor. Clínica médica. 2a ed. Barueri: Manole; 2016. v.1, p.648-66.

45. Task Force on the Management of Cardiovascular Diseases During Pregnancy of the European Society of Cardiology. Expert consensus document on management of cardiovascular diseases during pregnancy. Eur Heart J. 2003;24(8):761-81.

46. Siu SC, Colman JM, Sorensen S, Smallhorn JF, Farine $\mathrm{D}$, Amankwah KS, et al. Adverse neonatal and cardiac outcomes are more common in pregnant women with cardiac disease. Circulation. 2002;105(18):2179-84. https://doi. org/10.1161/01.CIR.0000015699.48605.08.

47. Elkayam U, Gleicher N, editors. Cardiac problems in pregnancy: diagnosis and management of maternal and fetal disease. 3rd ed. New York: Wiley-Liss; 1998.

48. Kampman MAM, Bilardo CM, Mulder BJM, Aarnoudse

Recebido em: 07.02.18

Aceito em: 04.04.18
JG, Ris-Stalpers C, van Veldhuisen DJ, et al. Maternal cardiac function, uteroplacental Doppler flow parameters and pregnancy outcome: a systematic review. Ultrasound Obstet Gynecol. 2015;46(1):21-8. doi: 10.1002/uog.14697.

49. Siu SC, Sermer M, Colman JM, Alvarez A. N, Mercier LA., Morton BC, et al. Prospective Multicenter Study of Pregnancy Outcomes in Women With Heart Disease. Circulation. 2001;104(5):515-21. https://doi.org/10.1161/hc3001.093437

50. Ouyang DW, Khairy P, Fernandes SM, Landzberg MJ, Economy KE. Obstetric outcomes in pregnant women with congenital heart disease. Int J Cardiol. 2010;144(2):195-9. doi: 10.1016/j.ijcard.2009.04.006.

51. Thorne S, MacGregor A, Nelson-Piercy C. Risks of contraception and pregnancy in heart disease. Heart. 2006;92(10):1520-5. doi: 10.1136/hrt.2006.095240

52. Regitz-Zagrosek V. ESC Guidelines on the management of cardiovascular diseases during pregnancy The Task Force on the Management of Cardiovascular Diseases during Pregnancy. Eur Hear. 2011;32(24):3147-97. doi: 10.1093/ eurheartj/ehr218.

53. Hebson C, Saraf A, Book WM. Risk Assessment and Management of the Mother with Cardiovascular Disease. Vol. 43, Clin Perinatol. 2016;43:1-22. doi: 10.1016/j. clp.2015.11.001

54. Ruys PTE, Hall R, Johnson MR, Webb G, Roos-Hesselink JW. Who classification as a risk predictor in pregnancy and heart disease. J Am Coll Cardiol. 2012;59(13):E843. doi: 10.1016/S0735-1097(12)60844-4.

55. Tedoldi CL, Freire C, Bub T, Sociedade Brasileira de Cardiologia. Diretrizes da Sociedade Brasileira de Cardiologia para Gravidez na Mulher Portadora de Cardiopatia. Arq Bras Cardiol. 2009;93(6 suppl.1):e110-78.

56. Azeka E, MB J, IB J, ES H, KC B, JD SN, et al. I Diretriz de Insuficiência Cardíaca (Ic) e Transplante Cardíaco, no feto, na criança e em adultos com cardiopatia congênita, da Sociedade Brasileira de Cardiologia. Arq Bras Cardiol. 2014;103(6 Suppl 2):1-126. http://dx.doi.org/10.5935/abc.2014S005

57. Ruys TPE, Roos-Hesselink JW, Pijuan-Domènech A, Vasario E, Gaisin IR, Iung B, et al. Is a planned caesarean section in women with cardiac disease beneficial? Heart. 2015;101(7):530-6. doi: 10.1136/heartjnl-2014-306497.

58. Levin H, LaSala A. Intrapartum obstetric management. Semin Perinatol. 2014;38(5):245-51. doi: $10.1053 / \mathrm{j}$. semperi.2014.04.013.

59. Bliacheriene F, Carmona MJC, Barretti C de FM, Haddad CMF, Mouchalwat ES, Bortolotto MR de FL, et al. Use of a minimally invasive uncalibrated cardiac output monitor in patients undergoing cesarean section under spinal anesthesia: report of four cases. Rev Bras Anestesiol. 2011;61(5):610-8, 334-8. doi: 10.1016/S0034-7094(11)70072-1. 